\title{
RADIP-JF - Radikalisierung Interventionsprogramm in der Jugendforensik
}

\author{
Leonardo Vertone ${ }^{1}$ \\ Eingegangen: 10. Februar 2021 / Angenommen: 19. Februar 2021 / Online publiziert: 16. März 2021 \\ (c) Der/die Autor(en) 2021
}

\section{Zusammenfassung}

Der Beitrag stellt mit dem RADIP ein psychotherapeutisches Interventionsprogramm vor, das zum Ziel hat, das Fortschreiten von Radikalisierungsprozessen bei Jugendlichen zu verhindern (Deradikalisierung) und die Wahrscheinlichkeit einer Ausübung extremistischer Taten zu verringern (Distanzierung). Das Programm ist deliktpräventiv konzipiert und vereint forensische und kognitiv-verhaltenstherapeutische Methodiken. Es ist strukturiert und multimodal und kann im Einzeloder im Gruppensetting durchgeführt werden. Nebst der Auseinandersetzung der Teilnehmenden mit Themen, die mit (ihrer) problematischen Radikalisierung in Verbindung stehen, beinhalten die Module Wissens- und Kompetenzzuwachs, Reflexion der eigenen Identität, Bewusstsein und Stärkung eigener prosozialer Ziele sowie Erhöhung der Entscheidungsund Steuerungsfähigkeit (ressourcenorientierter Zugang). Bestandteil des Programms ist eine Abklärungsphase, welche eine psychiatrisch-psychopathologische und (entwicklungs-)psychologische Diagnostik sowie ein forensisches ,risk assessment" beinhaltet. Das daraus resultierende Fallverständnis erlaubt es, individuelle Schwerpunkte zu setzen und flexibel zu intervenieren. Das Programm ist in einen multisystemischen und -disziplinären Rahmen eingebettet, d.h., Eltern oder sonstige nahe Bezugs- sowie Hilfspersonen werden miteinbezogen.

Schlüsselwörter Prävention · Therapie $\cdot$ Risikomanagement $\cdot$ Deradikalisierung $\cdot$ Jugendliche

\section{RADIP-JF-Radicalization intervention program in juvenile forensics}

\begin{abstract}
The RADIP is a therapeutic intervention program that aims to prevent radicalization processes (deradicalization) and to reduce the probability of committing acts of extremism (desistance). This intervention program follows the principals of crime prevention. It integrates forensic and cognitive-behavioral methods and is carried out in a single or group setting. Besides the focus on the process of radicalism the RADIP contains knowledge, understanding, implementation of techniques, personal skills, mindfulness, reflecting their own identity, awareness, competence, decision-making and controlling capabilities. Firstly, a concise diagnosis is made with respect to the psychopathology and developmental psychology including a risk assessment of the participants. Thereby it is possible to obtain an understanding of the underlying mechanism to apply individual interventions within the structure of the program. The program has a multisystemic approach; therefore, parents and other related persons are also involved.
\end{abstract}

Keywords Prevention $\cdot$ Therapy $\cdot$ Risk management $\cdot$ Deradicalisation $\cdot$ Juveniles

\section{Einleitung}

Leonardo Vertone, lic. phil.

leonardo.vertone@pukzh.ch

1 Klinik für Forensische Psychiatrie, Zentrum für Kinder- und Jugendforensik, Psychiatrische Universitätsklinik Zürich, Neptunstraße 60, 8032 Zürich, Schweiz
Angesichts des Aufkommens und der Erstarkung der Gruppierung Islamischer Staat (IS) und damit zusammenhängender Radikalisierungstendenzen bei Jugendlichen in Europa und in der Schweiz wurde im Zentrum für Kinderund Jugendforensik der Psychiatrischen Universitätsklinik 
Zürich 2015 das Interventionsprogramm RADIP entwickelt und angewendet.

Bei der Entwicklung des Programms wurde berücksichtigt, dass im Praxis- und im Forschungsdiskurs bezüglich Art und Intensität von Interventionen bei radikalisierten Jugendlichen mehrheitlich ein generalpräventiver, gesellschaftlicher und politischer und/oder ein spezialpräventiver sozialarbeiterischer Zugang als prioritär angesehen wird. Psychotherapeutische Zugänge finden sich seltener, obwohl psychiatrisch-psychologische Aspekte bei Radikalisierten diskutiert werden (Hoffmann 2017; Zick 2017; Fischer und Pfundmaier 2019; Leygraf 2019). Insbesondere bei Einzeltätern, die terroristische Aktivitäten ausüben, sollen psychische Störungen häufiger vorkommen als bei solchen, die in der Gruppe agieren (Radicalisation-Awareness-Network-H\&SC-Arbeitsgruppe 2017). Einig ist man sich darin, dass die Problematiken rund um Radikalisierungs- und Reintegrationsprozesse komplex und multikausal sind (Eser Davolio 2017; Eser Davolio und Villiger 2019; Logvinov 2019; Fischer und Pfundmaier 2019), und dass Interventionen dementsprechend differenziert, multimodal und mehrdisziplinär erfolgen sollen (Mansour 2015; El-Mafaalani et al. 2016; Duvillard 2019; Endrass et al. 2020). Auch das RADIP ist multimodal konzipiert und versteht sich als Teil eines breit angelegten Interventionskonzeptes.

Idealer- und typischerweise erfolgt die Durchführung des Programms im Auftrag einer strafjustiziellen (oder zivilrechtlichen) Behörde, welche die Teilnahme der Beteiligten sichert und allfällige weiterführende Maßnahmen umsetzt. In diesem Durchführungsprozess gilt es, die formellen und inhaltlichen Bedingungen rund um Auftragsklärung, Durchführung und Abschluss zu klären. Die Rechte und Pflichten aller Beteiligten in puncto Finanzierung, Berichterstattung, eingeschränkter Schweigepflicht, (Un-)Freiwilligkeit der Teilnahme, Informationsaustausch, Miteinbezug der Eltern und Dritter sind in einem Behandlungsvertrag zu klären. Der auftraggebenden Behörde ist in Form eines Berichtes Rechenschaft über den Erfolg des Programms abzulegen. Dieser Bericht beinhaltet eine abschließende Beurteilung und allfällige Empfehlungen für weiterführende Unterstützungs- und/oder Risikomanagementstrategien. Die Ergebnisse der Intervention werden in einer gemeinsamen Sitzung mit allen Beteiligten besprochen.

Grundsätzlich ist auch eine Durchführung des RADIP ohne „behördliches Korsett“ möglich. In solchen Fällen ergeben sich allerdings erfahrungsgemäß Schwierigkeiten in puncto Teilnahme und Finanzierung.

Das ambulant konzipierte Programm kann im Einzeloder im Gruppensetting durchgeführt werden. Während das Einzelsetting einen individuelleren Zugang ermöglicht, können im Gruppensetting entsprechende positive Effekte genutzt werden (Wittchen und Hoyer 2011). Im Gruppensetting ist allerdings darauf zu achten, die radi- kalisierungsfördernde Vernetzung und „Verbrüderung“ der Teilnehmenden zu vermeiden.

Das Programm ist strukturiert und multimodal. Je nach Fallverständnis sollen flexibel Tempo und Bearbeitungsintensität angepasst werden. Die Module sind innerhalb eines Spielraums von 10 bis 15 Sitzungen zu absolvieren.

Das Interventionsprogramm ist deliktpräventiv konzipiert und vereint kognitiv-verhaltenstherapeutische mit forensischer Methodik. Die konkrete Durchführung folgt üblichen kognitiv-verhaltenstherapeutischen Prinzipien (Rist et al. 2010; Wittchen und Hoyer 2011). Jugendlichengerecht wird möglichst visuell, auditiv, haptisch, mit einprägenden Übungen, Arbeitsblättern und mit webbasierten Inhalten gearbeitet.

Zielgruppe und Altersspanne: Das RADIP ist primär auf die Zielgruppe Jugendlicher (ca. 12 bis 18 Jahre) ausgerichtet. Es macht auch bei jungen Erwachsenen (18 bis 25 Jahre) Sinn, bei über 25-Jährigen nur in Einzelfällen.

Inhaltliche Indikation: Bei den Teilnehmenden müssen Radikalisierungsprozesse bzw. bereits ein gewisses Risiko festgestellt worden sein oder zumindest ein entsprechender Verdacht bestehen (kriminologisch sekundärpräventiver Ansatz). Kontraindikation besteht bei jeweils seit Kurzem aus Konflikt- oder gar Kriegsgebieten zurückgekehrten Personen und/oder bei final Radikalisierten (tertiärpräventiver Ansatz). Ebenso wenig sollen Personen teilnehmen, die sich rein vordergründig und strategisch mit der Thematik beschäftigen.

Das Programm wurde hinsichtlich einer islamistischdschihadistischen Radikalisierung entwickelt; es ist aber mit entsprechender Adaptation der informativ-edukativen Module für jegliche Form von Radikalisierung (Links-, Rechtsextremismus, Sekten u. Ä.) anwendbar.

Primäres Ziel ist es, das Fortschreiten von Radikalisierungsprozessen der Teilnehmenden zu verhindern (Deradikalisierung), die Wahrscheinlichkeit einer Ausübung extremistischer Taten zu verringern (Distanzierung) und ihre psychologisch-psychiatrischen Ressourcen hinsichtlich einer prosozialen Perspektive zu stärken (Ressourcenaktivierung). Daraus ergibt sich der gesellschaftsbezogene Nutzen der Opferprävention (Schutz der Bevölkerung) und der Verhinderung der Desintegration der Teilnehmenden (Senkung von Sozial- und Sicherungskosten). Der klientenbezogene Nutzen liegt darin, dass die Risiken der eigenen Fehlentwicklung, der Marginalisierung und Desintegration gesenkt werden sowie eigenes illegales Handeln und strafrechtliche Verfolgung, Haft und selbstschädigendes Verhalten verhindert werden (Schutz vor Selbstgefährdung). Im Gegenteil werden Wissen und Kompetenz, die eigene prosoziale Identität, Entscheidungs-, Steuerungsfähigkeit und Resilienz gestärkt und eine gesellschaftskonforme Perspektive aufgebaut. Der behördenbezogene Nutzen liegt darin, inhaltlich sinnvoll zur Prävention beizutragen und eine fachgerech- 
te, altersentsprechende psychiatrisch-psychologische sowie forensische Beurteilung zu gewährleisten, welche eine Orientierung im weiteren Umgang mit den politisch, gesellschaftlich und medial brisant diskutierten Fällen liefert.

\section{Abklärungsphase}

Einer jeden jugendforensischen Intervention geht ein deliktfokussiertes Assessment voraus (Bessler und Vertone 2017), und jede psychotherapeutische Intervention setzt eine Diagnostik voraus: Deshalb wird eine psychiatrisch-(entwicklungs-)psychologische Diagnostik mit einem auf die Radikalisierung und Ausübungsgefahr bezogenen ,risk assessment" kombiniert (Vertone 2017). Hierzu dienen das Studium vorhandener Akten, die Einholung von Informationen von Zuweisenden und bisher Beteiligten sowie mindestens ein Untersuchungsgespräch mit dem/r Klient*in und eines mit den Eltern.

In den Bereichen Radikalisierung und Ausübungsgefahr schwerwiegender Taten wurde in den letzten Jahren intensive Forschung betrieben, woraus einiges an empirischer Evidenz resultierte. Allerdings sind eine exakte Einordnung der Radikalisierung und Ausübungsgefahr (quantitatives Assessment) und eine distinkte Zuordnung zu Typen von Gefährder*innen (qualitatives Assessment) gerade im Jugendalter aufgrund der geringen Anzahl der Fälle und des entwicklungsdynamischen Charakters der Adoleszenz nicht abschließend möglich. Dennoch machen auf der Grundlage eigener praktischer Erfahrung und mehrerer Forschungsergebnisse und der Literatur in den Bereichen Radikalisierung und Bedrohungsmanagement (Böckler 2017; Hoffmann 2017; Hoffmann und Roshdi 2017) bzw. in Vereinigung und Adaptation verschiedener Arten der quantitativen und qualitativen Einteilung von Radikalisierungsstufen und -typologien (Endrass et al. 2015; Böckler et al. 2017; Eser Davolio 2019; Logvinov 2019) etwa die folgenden Orientierungsraster Sinn:

\section{Quantitative Einstufung}

Ordinalskalierte quantitative Einstufung von Radikalisierung und deren Bedeutung hinsichtlich der Anwendung des RADIP:

Radikalisierungsstufe 1, „grün“ und „gelb“ Zufällig mit der Thematik in Berührung. Verhalten weder substanziell noch strafrechtlich relevant $\rightarrow$ RADIP je Verhältnismäßigkeit sinnvoll.

Radikalisierungsstufe 2, "gelb“, "orange“ Fortgeschrittene Beschäftigung, strafrechtlich relevant, Teileinbettung in übliche schulische, berufliche und soziale Strukturen sowie
Ambivalenzen vorhanden $\rightarrow$ Hauptgruppe des Programms, RADIP indiziert.

Radikalisierungsstufe 3, "rot“ Radikalisierte Rückkehrer aus einschlägigen Gebieten und/oder aus entsprechenden Gruppierungen $\rightarrow$ RADIP nicht indiziert, als ergänzende Hilfeleistung z. T. sinnvoll.

\section{Qualitative Zuordnung zu Radikalisierungstypen}

Eine Zuordnung zu Radikalisierungstypologien macht diagnostisch und im Hinblick auf die Durchführung des RADIP Sinn. Sie ermöglicht eine Orientierung, wie innerhalb der vorgegebenen Struktur des Programms individuell vorzugehen ist:

Typ 1 „,der Unsichere“ Beeinflussbar, soziale Zugehörigkeit und Orientierung suchend, Wunsch nach Anerkennung. Gewalttätig innerhalb der Gruppierung und deren Dynamiken. Ideologie als Mittel zum Zweck, zugehörig zu sein. $\rightarrow$ RADIP erfolgversprechend durch Ressourcenorientierung.

Typ 2 „der Persönlichkeitsgestörte“ a) Dissozial: Normüberschreitungen und Gewaltanwendung und -legitimation normal, häufig impulsiv, aufbrausend und bereits straffällig. Ideologie unwichtig, wird zur Legitimierung von Gewalttaten genutzt. Motto: „Hauptsache Gewalt“ $\rightarrow$ RADIP teils erfolgversprechend durch forensischen Zugang. b) Narzisstisch: genießt Bedeutung und Aufwertung innerhalb der Gruppe und Außenwirkung der Radikalisierung. Identifiziert sich stark mit der Aufgabe bzw. der Ideologie und stabilisiert sein Selbstwertgefühl damit. $\rightarrow$ RADIP teils erfolgversprechend durch psychotherapeutischen Zugang.

Typ 3 "der Ideologe“ Adaptiert sich kontextspezifisch an gesellschaftliche Regeln und Normen, kann sich anpassen, in der Regel keine strafrechtlichen Auffälligkeiten, seine Norm gilt, „Wir-vs.-sie“-Haltung, Andersgläubige sind illegitim und verdienen negative Konsequenzen, ist unabhängig von Gruppierung, ,Schläfer“, langer Verfestigungsprozess. Ideologie und Radikalisierung zentral und verankert. $\rightarrow$ RADIP wirkungslos bis kontraindiziert, allenfalls hinsichtlich einer Distanzierung zweckmäßig.

Typ 4 "der psychisch Kranke“ Prosoziale Regeln und Normen verankert, Realitätswahrnehmung und/oder Steuerungsfähigkeit durch psychische Störung getrübt. Gewalt innerhalb von „Krankheitsschub“, lässt sich in psychisch besonders labilen Phasen von ungünstigen ideologischen Dynamiken mitreißen. Ideologie nicht bedeutsam, phasenweise und krankheitsbezogen aber sehr wohl handlungsleitend. $\rightarrow$ RADIP erfolgversprechend i. S. psychiatrisch- 


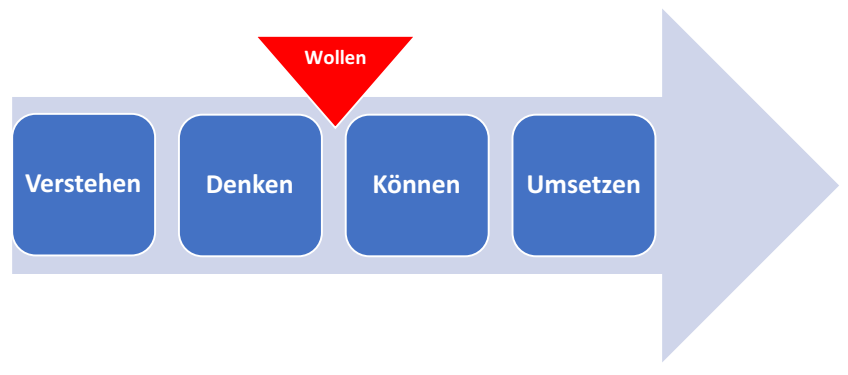

Abb. 1 Übergeordnetes Prinzip und Ablauf der Interventionsphase

psychotherapeutischer Einordnung, Behandlung und $\mathrm{Zu}$ weisung, kontraindiziert bezüglich Moduldurchführung.

\section{Interventionsphase}

Die Module der Interventionsphase sind chronologisch den in Abb. 1 abgebildeten Phasen zugeordnet.

Modul "Gesetzliche Grundlagen“ Die Auseinandersetzung mit der gesetzlichen Grundlage und den (möglichen) strafrechtlichen Konsequenzen von Radikalisierung bzw. entsprechendem Verhalten dient den Teilnehmenden, sich über das Ausmaß und die rechtliche Bedeutung des eigenen, zuweilen unüberlegten bis fahrlässigen Verhaltens (gerade auf Social Media) bewusst zu werden. Die Auseinandersetzung mit den konkreten Gesetzestexten kann bereits abschreckende Wirkung haben.

Modul "Ideeller Hintergrund, Absicht und Rekrutierungsstrategien der Gruppierung" Die Auseinandersetzung mit dem ideellen Hintergrund der jeweiligen Gruppierung fördert oftmals Aha-Effekte zu Tage, indem dem Teilnehmenden bewusst wird, dass dieser sich gar nicht mit eigenen - bisher meist ohnehin nicht groß reflektierten Vorstellungen und Motiven deckt. Die konkrete Nennung der oftmals kruden Überzeugungen oder Gewaltabsichten solcher Gruppierungen, wie z.B. deren soziale, gesellschaftspolitische und/oder regionale (Besitz-)Ansprüche, kann bereits auf- und abschrecken und regt zumindest einen Diskurs über Sinn und Unsinn derselben an.

Die Wissensvermittlung über die Gefahren der Zugehörigkeit zur Gruppierung, zu deren Rekrutierungsstrategien, zum Beeinflussungs-, Manipulations- und Propagandaverhalten stärkt die Fähigkeit der Teilnehmenden, sich ebensolchen zu entziehen. Um ein autonomes und (differenzierteres) Nachdenken über die Thematik anzuregen, die Ambiguitätstoleranz und Toleranz für andere Meinungen der Teilnehmenden zu fördern, lohnt es sich, möglichst nüchtern und deskriptiv zu bleiben und im Sinne des Sokratischen Dialogs Fragen aufzuwerfen. So werden Denkanstöße erbracht, und es entstehen Diskussionen, z. B. über das Wesen (die Vorzüge) demokratischer Staatsformen und humanistischer Werte. Idealerweise entstehen ein Hinterfragen ungünstiger Narrative und der Aufbau günstiger (Gegen-)Narrative. Demgegenüber führen frühe offensichtliche, parteiische und moralische Appelle eher zu unproduktiver Reak$\operatorname{tanz}$.

Modul „Eigene Radikalisierungsgeschichte“ Die Ausarbeitung ihrer individuellen Radikalisierungsgeschichte soll den Teilnehmenden dazu dienen, die eigene (Fehl-)Entwicklung auf kognitiver, emotionaler und Verhaltensebene zu verstehen. Die Bedeutung eigener Anteile (interne Risikofaktoren) sowie die Rolle von Umweltbedingungen (externe Risikofaktoren) wird eruiert. Diese radikalisierungsbezogene Biografiearbeit ist ein zentraler Bestandteil des RADIP und dient auch den Therapeut*innen, ein erweitertes Fallverständnis aufzubauen. Diesem Modul sollte viel Raum gegeben werden. Es setzt Vorkenntnisse seitens der Therapeut*innen voraus. Den Teilnehmenden den eigenen qualitativen und quantitativen Radikalisierungstyp/-grad zu vergegenwärtigen, ist ebenfalls Teil dieses Moduls.

Module „Identität" und „Ziele und Ressourcen“ Die Auseinandersetzung mit der eigenen Identität und mit eigenen Zukunftsvorstellungen soll das Bewusstsein der Teilnehmenden darauf (zurück-)lenken, dass sie ,eigentlich wie alle anderen“ prosoziale Bedürfnisse (,anständiger Mensch“, „Platz in der Welt“, „Zufriedenheit“, „Bindung“) haben, dass sie die meiste Zeit des Lebens prosozial identifiziert waren und/oder sein wollen und viele Eigenschaften und Beziehungen besitzen, die eine solche prosoziale Identität (er-)füllen. Es gilt, ressourcenorientiert in den verschiedenen Lebensbereichen (Familie, Freunde, Hobbys/ Interessen, Partnerschaft, Schule/Beruf) prosoziale Aspekte zu finden und sich auf die Suche nach positiven Aspekten in der Vergangenheit, Gegenwart und Zukunft zu machen. Die persönliche Priorität prosozialer Ziele und Pläne wird erhöht, eine prosoziale Perspektive gefördert. Eigene Fähigkeiten, Stärken und Ressourcen werden gesammelt. Denkprozesse in Richtung konkreter und realistischer Umsetzungsmöglichkeiten und Zwischenschritte werden angeregt. Zuversicht zur Zielerreichung wird erhöht.

Modul „Bilanzierung - Vor- und Nachteile von Radikalisierung" Die vorangegangenen Module bilden die Grundlage für dieses motivational wichtige Modul. Prosoziale Denkprozesse und Überzeugungen sind angeregt worden, sodass die in diesem Modul aktivierte Gegenüberstellung von Vor- und den Nachteilen einer anhaltenden radikalisierten Lebensführung mit denjenigen einer prosozialen Lebensführung nicht mehr allzu einseitig zugunsten der Radikalität ausfallen sollte. Es soll den Teilnehmenden ersichtlich werden, welche konkreten negativen Auswirkungen das ei- 
gene radikale Verhalten auf die Erfüllbarkeit der persönlichen Ziele hat. Idealerweise kommen die Teilnehmenden in der Erarbeitung der verschiedenen Aspekte von alleine darauf, dass den kurzfristigen Vorteilen einer ebensolchen Lebensführung (Zugehörigkeitsgefühl, Bedeutung, Flucht vom überfordernden Alltag usw.) stärker zu gewichtende langfristige Nachteile (anhaltende Registrierung und Verfolgung seitens der Behörde, Vertrauensverlust der Eltern, berufliche Einschränkungen, keine Ausbildung, Unfreiheit, Desintegration, Isolation, Unzufriedenheit, gesellschaftlicher Schaden, ständiger Stress usw.) gegenüberstehen. Und dass es für sie also wichtig ist, alles daran zu setzen, aus der Radikalisierungsschlaufe auszusteigen, bzw. dass sich umgekehrt eine Besinnung auf den kurzfristig mühsam erscheinenden prosozialen Weg (Überforderung, Anstrengung, Verletzlichkeit, Schulprobleme usw.) langfristig lohnt (,Frau \&Kind“, „Geld und Auto“, ein stressfreies Leben). Genereller geht es in diesem Modul um die Förderung konsequenzenorientierten Denkens.

Modul "Wollen - Mein Entschluss" Dieses Modul markiert den Abschluss der Klärungsarbeit. Durch Anregung eines motivationalen Prozesses gemäß den ,stages of change“ im Transtheoretischen Modell von Prochaska und DiClemente (Schaffer 2013) wird eine bewusste Entscheidung für (oder gegen) eine Verhaltensänderung angeregt. Dieser Prozess wird unmittelbar mit dem vorangegangenen Bilanzierungsprozess verbunden, in dem alle bisher gesammelten relevanten Faktoren bewusst gemacht werden. In der Regel gelingt es den Teilnehmenden im Alltag nicht, alle Aspekte gleichzeitig zu berücksichtigen. Relevante (präventive) Faktoren werden ausgeblendet. Dementsprechend sind gefährdete Jugendliche anfällig für einschlägige Beeinflussung radikalisierter Personen und Gruppierungen, die einzelne Aspekte einseitig in ihrem Sinne betonen. Im vorangegangenen Bilanzierungsmodul wurden aber in breit gefächerter Manier alle Faktoren gesammelt, sodass die Teilnehmenden sie in diesem Modul in ihren Entscheidungsprozess für oder gegen künftige Radikalisierung einbeziehen können. Die Willensentscheidung wird betont markiert, was das Erleben der Eigenverantwortung fördert. Die Motivation für die nachfolgenden bewältigungsorientierten Module wird gestärkt.

Idealerweise erfolgt ein klarer Entschluss zur prosozialen Lebensführung, begleitet von Zuversicht, Lust und Durchhaltewillen, neuen Vorbildern und eigenen Denkmodellen nachzueifern sowie attraktive Zukunftsperspektiven und -pläne zu verfolgen. In der klinischen Realität stellt dieser Entscheidungsprozess bei der einschlägigen - im ungünstigen Fall lediglich extrinsisch motivierten Klientel eine besondere Herausforderung dar, insbesondere in knapp bemessener Zeit und/oder bei hoher Zahl von Teilnehmenden. Nicht immer explizieren die Teilnehmenden ihre echten Absichten im prosozialen Sinn.
Einige formulieren hehre Absichten nur vordergründig. Da die Motivations- und Klärungsarbeit in der Regel nicht komplett abgeschlossen werden kann, sind klärende Interventionen auch später, in den bewältigungsorientierten Modulen, notwendig. In besonders hartnäckigen Fällen muss die Phase der motivationalen Entscheidung erneut bzw. länger prozessual aktiviert werden. Im Falle einer Ablehnung eines Bekenntnisses zur Haltungs- und Verhaltensänderung ist dies zumindest im Sinne der erweiterten Diagnostik erkenntnisreich. In den meisten Fällen ergeben sich aber erfahrungsgemäß zumindest Ambivalenzen, an denen angesetzt werden kann.

Modul "Meine Risikofaktoren und -situationen“ In diesem Modul werden die Erkenntnisse aus dem Modul „Eigene Radikalisierungsgeschichte“ verdichtet, und es werden daraus konkrete individuelle Risikofaktoren/-situationen bestimmt. In einer Risikosituation ist die Chance höher, sich auf radikale und extremistische Ideen zu berufen, sich verleiten zu lassen, die Radikalität zu vertiefen oder gar Gewalt anzuwenden. Dazu können nebst eigenen psychopathologischen oder Persönlichkeitsmerkmalen auch externe Risikosituationen (z.B. mit Kollege X), -dynamiken (z.B. in einschlägigen Gruppenveranstaltungen) oder auch Lebensphasen (z. B. Arbeitslosigkeit) gehören.

Häufig ähneln Risikosituationen denjenigen, die dazu führten, dass das Interventionsprogramm angeordnet wurde.

Modul „Persönliche Handlungspläne“ Handlungspläne sind das Destillat der Intervention. Gelingt es einem Teilnehmer nicht, seine Einsichten und Fertigkeiten umzusetzen, bleiben die bisher theoretisch erarbeiteten Veränderungen wirkungslos. Gute Absichten, Kenntnis von Risikofaktoren und -situationen genügen $u$. U. nicht. Nicht nur müssen sich Teilnehmer des Programms im entscheidenden Moment ihrer Verantwortung gewahr werden und einen klaren Entschluss zur aktiven Verhaltensänderung fassen; sie sollten auch über die Fertigkeit verfügen, sich entsprechend präventiv verhalten zu können. Hierzu werden konkrete, einfache, einprägsame und realistisch umsetzbare präventive Handlungspläne und -strategien und Alternativlösungen erarbeitet, um Risikosituationen a) zu verhindern und zu vermeiden oder sich b) diesen, die oft von hohem Stress und gruppendynamischen Prozessen geprägt sind, zu entziehen, falls sie nicht verhindert werden konnten.

Modul "Training“ Im Rahmen des RADIP ist kein komplettes Training sozialer oder emotionaler Kompetenzen oder anderweitiger Art (je Radikalisierungstyp) möglich. Primär geht es hier um Psychoedukation bezüglich entsprechender Trainings und die Stärkung der Einsicht, dass ebensolche den Teilnehmenden einen Mehrwert hinsichtlich ihrer 
Ziele bringen können. Ein Commitment hinsichtlich allfällig indizierter weiterführender Trainings und Interventionen wird angestoßen. Sehr wohl soll man allerdings die Kraft von selbst kurzen kognitiv-verhaltenstherapeutischen Übungen, gerade in der Gruppe, nicht unterschätzen. Rollenspiele zum Zwecke des Praxistransfers der erarbeiteten Handlungspläne, effektive Anweisungen bezüglich Abgrenzung, Stärkung einzelner Fähigkeiten wie autonomes Denken, soziales Vernetzungstraining, Selbstwertstärkung, selbstsicheres Auftreten, Entscheidungstraining, gewaltfreies Problemlösen, angemessene Selbstbehauptung u. Ä. können sehr effizient sein.

\section{Nachsorge - Transfer und Verankerung}

Modul „Umsetzung“ Über das Trainieren und Einüben der Strategien im Therapieraum hinaus sollen die Teilnehmenden aus ihrem konkreten Leben berichten bzw. bestenfalls bereits einschlägige Situationen bewältigen. Zudem geht es darum, erarbeitete Alternativen, Unterstützungsangebote oder positive Rollenvorbilder konkret umzusetzen (Telefonnummer wählen, Adresse aufsuchen, Person X ansprechen). Hausaufgaben und Absprachen mit den Eltern stärken diesbezüglich die Verbindlichkeit.

"Einbezug der Eltern" Jugendliche sind beeinflussbar, im ungünstigen wie im günstigen Sinn. Je besser die prosoziale und familiäre Einbettung, umso kleiner die Wahrscheinlichkeit der Radikalisierung. Zur Festigung der Nachhaltigkeit des Praxistransfers werden die Eltern in die Verantwortung genommen, ihrerseits die prosoziale Vernetzung ihres Schützlings zu unterstützen, die Anwendung von präventiven Strategien zu unterstützen. Hierzu werden sie zum Gespräch eingeladen, ohne sowie mit ihren Schützlingen. Der Einbezug der Eltern (oder sonstigen nahen Bezugspersonen) ist wichtig, da dadurch wichtige bindungsbezogene Gefühle (re-)aktiviert werden können, was die Entfremdung, Desintegration verhindern kann und die Hemmschwelle für zerstörerische Handlungen erhöht. Emotionale Familien- und bindungsbezogene Gegennarrative können gegen spaltende und separierende extremistische Anschauungen wirken, um erste oder weitere, z. T. nur brachliegende Zweifel bei den Jugendlichen zu reaktivieren oder zu verstärken.

Die familiären Hintergründe sind verschieden. Die Jugendlichen kommen meist aus ,,normalen Familien“ aus allen Schichten. Die allermeisten Eltern teilen die radikalen Ansichten der Kinder nicht, erst recht wünschen sie ihnen nicht die damit zusammenhängenden potenziellen Konsequenzen. Jeder Fall ist individuell, wobei sich die meisten Jugendlichen in einem Teufelskreis wiederfinden, aus persönlicher Krise, Isolation, Abwendung von Zuhause, Kon- flikten mit den Eltern. Das Gespräch mit den Eltern dient somit auch dazu, diese zu stärken, in ihrem Bemühen um ihre Kinder, anstatt dass sie sich überfordert von diesen abzuwenden.

Modul „Übergabe an Behörde und Nachsorge“ Der Programmverlauf und die daraus gewonnenen Erkenntnisse werden in einem Bericht an die Behörde festgehalten. Nebst der psychiatrisch-psychologischen erfolgen die finale forensische Beurteilung (Radikalisierung, Gefährlichkeit) und entsprechende Empfehlungen für allfällige weiterführende Maßnahmen. Die Resultate werden in einer systemischen Abschlusssitzung allen Beteiligten eröffnet.

Im Rahmen der Intervention können bedeutsame Veränderungsprozesse in Gang gebracht werden, die sich selbst genügen können. Bei Fällen mit erheblichen Fehlentwicklungen, schwereren Psychopathologien, verfestigten Ideologien und/oder tiefgreifender Verwahrlosung können aber in der abgesteckten Zeit keine nachhaltigen Veränderungen erreicht werden. In solchen Fällen dient das RADIP als Grundlage für weiterführende Interventionen in den Bereichen Erziehung, soziale und berufliche Integration, Psychotherapie und/oder Risikomanagement. So können je Fallverständnis etwa beim unsicheren Typus weitere psychotherapeutische Bemühungen, Training sozialer Kompetenzen oder integrative pädagogische Maßnahmen, beim dissozialen Typus forensisch-deliktpräventive Therapien und/ oder sichernde Maßnahmen, beim ideellen Typus sichernde Maßnahmen und Integration in gemäßigte Kreise und beim psychisch Kranken die psychiatrische Versorgung bzw. die störungsspezifische Behandlung empfohlen werden, oder es kann bei allen Typen eine Familientherapie Sinn machen.

Da Rückfälle innerhalb einer sensiblen Phase der ersten ca. 12 Monate (exemplarisch: Veismann et al. 2019) nach jeweiliger Intervention wahrscheinlicher sind, erfolgt 3 bis 6 Monate nach Beendigung des Programms eine Auffrischungssitzung.

\section{Möglichkeiten und Grenzen des Programms}

Im Umgang mit radikalisierten Jugendlichen ist multidisziplinär und -systemisch vorzugehen. Auch wenn das RADIP dieser Tatsache durch Einbezug von Eltern und Behörden Rechnung trägt, ist der Fokus forensisch-psychotherapeutisch: Die psychische Gesundheit, Entwicklung und Persönlichkeit, familiäre Situation werden eruiert und auf der Basis psychologisch-psychiatrischer und kriminologischer Risikofaktoren die Gefahr externalisierender Gewalt eingeschätzt. Daraus werden forensisch-psychotherapeutische Interventionen und Empfehlungen für ein weiterführendes Risikomanagement abgeleitet. Aufgaben, die sozialarbeiterisch, schul- und berufsintegrativ, erzie- 
herisch oder sichernd-sanktionierend sind, können nicht übernommen werden. Zudem können weder eine exakte $(, 80 \%$ ") noch eine kategoriale (,Ja/Nein“-)Risikobeurteilung erfolgen. Ideologien können nicht ,wegtherapiert“ und Radikalisierte in kurzer Zeit und ohne ein multidisziplinäres Helfersystem nicht entradikalisiert werden. Radikalisierung und Entradikalisierung sind langfristige Prozesse. Die Grundidee des RADIP ist dementsprechend, eine klar definierte Teilaufgabe in einem komplexen und längerfristigen Prozess zu übernehmen, innerhalb einer relativ kurzen Zeit intensiv zu intervenieren und dann wieder an das mehrdisziplinäre Nachsorgenetzwerk (Eltern, Gemeinde, Schule, Arbeitsstelle, Behörde, Sozialarbeit, Polizei, Anwaltschaft, Imame u. Ä.) zu übergeben.

Seit der Entwicklung des Programms führten wir das Programm entgegen den Erwartungen von uns und den Behörden lediglich in 2 Fällen vollständig und in weiteren 2 Fällen mit vereinzelten Modulen durch. Ein Wirkungsnachweis ist damit nicht erbracht. Hierzu bedürfte es größerer Gruppen und einer Evaluation der weiteren Verläufe. Mögliche Gründe für die geringe Anzahl könnten sein, dass die Jugendlichen, die in die Hauptzielgruppe des Programms fallen würden, nicht angemeldet werden, da die Teilnahme an einem Programm eines hochspezialisierten Zentrums in einem Zwangskontext den Behörden evtl. nicht verhältnismäßig erscheint. Zudem werden viele Jugendliche der Radikalisierungsstufe 1 offenbar (es liegen uns hier keine verlässlichen Informationen vor) in den bereits vorhandenen familiären und schulisch-beruflichen Strukturen oder sonstigen Anlaufstellen von stärkerer Radikalisierung abgehalten. Angemeldet werden mehrheitlich die Fälle, bei denen eine Radikalisierung bereits zu verfestigt ist. Für solche Fälle ist das vorliegende Interventionsprogramm zu niederschwellig, weshalb ebenfalls keine Durchführung erfolgt. Eine Durchmischung der Hoch- und Niedrigrisikogruppen ist gerade bei dieser Thematik nicht zu empfehlen. Letztlich kann unsere praktische Erfahrung aber auch darauf hinweisen, dass die tatsächliche Anzahl von Risikopersonen möglicherweise tiefer ist, als es die mediale Berichterstattung nahelegt.

Funding Open access funding provided by University of Zurich

Interessenkonflikt L. Vertone gibt an, dass kein Interessenkonflikt besteht.

Open Access Dieser Artikel wird unter der Creative Commons Namensnennung 4.0 International Lizenz veröffentlicht, welche die Nutzung, Vervielfältigung, Bearbeitung, Verbreitung und Wiedergabe in jeglichem Medium und Format erlaubt, sofern Sie den/die ursprünglichen Autor(en) und die Quelle ordnungsgemäß nennen, einen Link zur Creative Commons Lizenz beifügen und angeben, ob Änderungen vorgenommen wurden.

Die in diesem Artikel enthaltenen Bilder und sonstiges Drittmaterial unterliegen ebenfalls der genannten Creative Commons Lizenz, sofern sich aus der Abbildungslegende nichts anderes ergibt. Sofern das be- treffende Material nicht unter der genannten Creative Commons Lizenz steht und die betreffende Handlung nicht nach gesetzlichen Vorschriften erlaubt ist, ist für die oben aufgeführten Weiterverwendungen des Materials die Einwilligung des jeweiligen Rechteinhabers einzuholen.

Weitere Details zur Lizenz entnehmen Sie bitte der Lizenzinformation auf http://creativecommons.org/licenses/by/4.0/deed.de.

\section{Literatur}

Bessler C, Vertone L (2017) Deliktorientierter Behandlungsansatz. In: Bilke-Hentsch O, Sevecke K (Hrsg) Aggressivität, Impulsivität und Delinquenz. Thieme, Stuttgart, S 158-162

Böckler N (2017) Der sog. Islamische Staat und die Mudschaheddin aus dem Westen: Radikalisierungsprozesse unter schwarzer Flagge. In: Böckler N, Hoffmann J (Hrsg) Radikalisierung und extremistische Gewalt. Verlag für Polizeiwissenschaft, Frankfurt, S 27-48. ISBN 978-3-486-70402-0

Böckler N, Allwin M, Hoffmann J, Zick A (2017) Früherkennung von islamistisch motivierter Radikalisierung - Vorstellung und empirische Validierung eines verhaltensbasierten Instrumentes zum Fallscreening. Kriminalistik 8-9:491-497

Duvillard A (2019) Prävention von Radikalisierung und gewalttätigem Extremismus als interdisziplinäre Verbundsaufgabe. In: Schwarzenegger C, Brunner R (Hrsg) Bedrohungsmanagement. Schulthess, Zürich, S 7-12. ISBN 978-3-7255-8070-5

El-Mafaalani A, Fathi A, Mansour A, Müller J, Nordbruch G, Waleciak J (2016) Ansätze und Erfahrungen der Präventions- und Deradikalisierungsarbeit (HSFKReport Nr. 6/2016). In: Biene J, Daase C, Gertheiss S, Junk J, Müller H (Hrsg) HSFK-Reportreihe „Salafismus in Deutschland“. Frankfurt am Main. Hessische Stiftung Friedens- und Konfliktforschung. ISBN 978-3-946459-02-6. https://nbn-resolving.org/urn:nbn:de:0168-ssoar-46683-7

Endrass J, Sadowksi F, Böckler N, Rossegger A (2015) Der Weg zum (terroristischen) Attentäter: Gewalt legitimieren, um Gewalt auszuüben. Kriminalistik 5:328-334

Endrass J, Weber M, Rossegger A (2020) Begutachtung im Bereich des Extremismus. In: Psychiatrische Begutachtung: ein praktisches Handbuch für Ärzte und Juristen, 7. Aufl. Elsevier, München, S 831-837 https://doi.org/10.1016/B978-3-437-22903-9.000475. ISBN 978-3-437-22903-9

Eser Davolio M (2017) Jihadistische Radikalisierung in der Schweiz eine Aufgabe für die Soziale Arbeit? Migrat Soz Arb 3:1432-6000

Eser Davolio M (2019) Background of Jihadist radicalisation and measures for prevention and intervention in Switzerland. Sozialpolitikch. https://doi.org/10.18753/2297-8224-144

Eser Davolio M, Villiger C (2019) Exploring the pathway from radicalisation to disengagement: comparison of dissonances experienced by a Jihadi foreign fighter and a right-wing extremist. Sozialpolitikch Vol. https://doi.org/10.18753/2297-8224-141

Fischer MV, Pfundmaier M (2019) Die Psychologie der terroristischen Radikalisierung: Wie sich Menschen radikalisieren und Prävention gestaltet werden kann. Psychol Rundsch 70:138-141. https:// doi.org/10.1026/0033-3042/a000440

Hoffmann J (2017) Bedrohungsmanagement und psychologische Aspekte der Radikalisierung. In: Böckler N, Hoffmann J (Hrsg) Radikalisierung und extremistische Gewalt. Verlag für Polizeiwissenschaft, Frankfurt, S 71-96. ISBN 978-3-86676-478-1

Hoffmann J, Roshdi K (2017) Bedrohungsmanagement - Projekte und Erfahrungen aus der Schweiz. Verlag für Polizeiwissenschaft, Frankfurt. ISBN 978-3-86676-332-6

Leygraf N (2019) Zur Phänomenologie islamistischer Straftäter. In: Schwarzenegger C, Brunner R (Hrsg) Bedrohungsmanagement. Schulthess, Zürich, S 31-42. ISBN 978-3-7255-8070-5

Logvinov M (2019) Risikobewertung extremistischer Gewalt. Springer VS, Wiesbaden https://doi.org/10.1007/978-3-658-25123-9 
Mansour A (2015) Generation Allah - Warum wir im Kampf gegen religiösen Extremismus umdenken müssen. Fischer, Frankfurt am Main. ISBN 978-3-10-002446-6

Radicalisation Awareness Network H\&SC Arbeitsgruppe (2017) Risikobewertung rund um Einzelakteure, Ex-Post-Beitrag. Mechelen. https://ec.europa.eu/home-affairs/sites/homeaffairs/files/whatwe-do/networks/radicalisation_awareness_network/about

Rist F, Witthöft M, Bailer J (2010) Grundlagen der kognitiven Verhaltenstherapie. In: Arolt V, Kersting A (Hrsg) Psychotherapie in der Psychiatrie. Springer, Berlin Heidelberg, S 45-72

Schaffer JA (2013) Stages-of-change model. In: Gellman MD, Turner JR (Hrsg) Encyclopedia of behavioral medicine. Springer, New York, S 121-136 https://doi.org/10.1007/978-1-4419-10059_1180

Veismann S, Knecht G, Tozdan S, Briken P (2019) Legalbewährung nach einer Entlassung aus dem Maßregelvollzug ( $\$ 63 \mathrm{StGB}$ ) in der Metropolregion Hamburg. Forens Psychiatr Psychol Kriminol 13:343-354. https://doi.org/10.1007/s11757-019-00566-Z

Vertone L (2017) Radikalisierte Jugendliche - Abklärung und Intervention. Vortrag an der Tagung „Radikalisierte Jugendliche - eine Herausforderung auch für die Jugendstrafrechtspflege" der Oberjugendanwaltschaft Kanton Zürich und Bundesanwaltschaft, 31. Okt. 2017

Wittchen H-U, Hoyer J (2011) Klinische Psychologie und Psychotherapie. Springer, Berlin Heidelberg

Zick A (2017) Extremistische Inszenierungen: Prozesse der Radikalisierung und ihre Prävention. In: Böckler N, Hoffmann J (Hrsg) Radikalisierung und extremistische Gewalt. Verlag für Polizeiwissenschaft, Frankfurt, S 3-26. ISBN 978-3-86676-478-1 